\title{
Gefährliche Fehllenkung durch Wirtschaftlichkeitsverfahren
}

\author{
Michel Romanens ${ }^{a}$, Flavian Kurth ${ }^{b}$, Walter Warmuth ${ }^{c}$ \\ ${ }^{a}$ Dr. med., Präsident VEMS; ${ }^{b}$ Sekretär VEMS; ${ }^{c}$ Dr., Gesundheitsforen Leipzig
}

Es gilt unter Experten heute als erkannt, dass eine zu schnelle medikamentöse Behandlung psychischer Krankheiten wie Depressionen und Angststörungen bei leichter Erkrankung zu einer Chronifizierung, im schlimmsten Fall zu einer Invalidisierung der Patienten führen kann, was neben vermeidbarem Leid auch zu vermeidbaren Kosten führt. Umso mehr erstaunt es, dass ausgerechnet die Wirtschaftlichkeitsverfahren von santésuisse diese Fehlentwicklung fördern. Wie der Artikel an einem besonders deutlichen Beispiel aufzeigt, bleibt den behandelnden Ärzten oft nur ein solches Verhalten, um ihre Durchschnittskosten so zu halten, dass sie in der Statistik nicht als auffällig erscheinen. Trotz vorgängig eingeholter und von den Versicherern bewilligter Kostengutsprachen werden Ärzte, die psychotherapeutische Massnahmen sinnvoll anwenden und Psychopharmaka nur dort, wo sie tatsächlich angezeigt sind, dafür belangt und zum Zahlen von Vergleichen gedrängt. Die Verbindung der psychiatrisch-psychotherapeutisch tätigen Ärztinnen und Ärzte der Schweiz FMPP hat das Problem er-

\section{Résumé}

Les experts s'accordent aujourd'hui sur le fait qu'un traitement médicamenteux trop rapide de maladies psychiques telles que la dépression et les troubles anxieux peut, en cas d'affection légère, entraîner une chronicisation ou, dans le pire des cas, une invalidité des patients, ce qui, outre des souffrances évitables, génère aussi des coûts évitables. Que ce soient précisément les procédures d'économicité de santésuisse qui favorisent cette évolution défavorable ne peut donc que surprendre. Comme le montre l'article en s'appuyant sur un exemple particulièrement flagrant, les médecins traitants n'ont souvent pas d'autre choix que d'adopter un tel comportement pour que leurs coûts ne paraissent pas anormaux dans les statistiques. Bien qu'ils aient au préalable obtenu des garanties de prise en charge des frais approuvées par les assureurs, les médecins qui emploient des mesures de psychothérapie judicieuses et ne recourent aux médicaments psychotropes que lorsque ceux-ci sont réellement indiqués sont poursuivis et contraints de payer des comparaisons. La Fédération suisse des médecins psychiatres-psychothérapeutes (FMPP) est consciente du problème, mais ne s'impose pas face à santésuisse. kannt, setzt sich gegenüber santésuisse aber nicht durch.

\section{Gesprächstherapie versus Psycho- pharmaka}

Die Behandlungsempfehlung bei leichter bis mittelschwerer Depression lautet, auf Psychotherapie zu setzen und vom Einsatz von Psychopharmaka möglichst abzusehen [1]. Es ist von den Fachgesellschaften und Experten weithin erkannt, dass so einer Chronifizierung der Krankheit oder gar einer Invalidisierung der betroffenen Patienten entgegenzuwirken ist, was Leid verhindert und Kosten spart. Die Praxis sieht allerdings anders aus. In seinem Artikel «Den Therapeuten am Draht» [2] im Tages-Anzeiger vom 6. Februar 2017 moniert Felix Straumann denn auch richtig: «Ärzte behandeln leichte Depressionen meistens mit Psychopharmaka statt mit Psychotherapie, wie es die Richtlinien empfehlen würden.» Dem Grund für dieses Fehlverhalten ist der Autor auch auf der Spur, wenn er weiter schreibt: «Zudem erfordert die Behandlung durch einen Psychotherapeuten Zeit und ist auf den ersten Blick teuer.» Allerdings geht Herr Straumann den Ursachen hierfür nur oberflächlich nach, wenn er diese in der mangelnden Bereitschaft der Patienten und in den langen Wartezeiten für eine Psychotherapie ausmacht. Wo ein Bedarf besteht, da bildet sich ja in der Regel auch ein Angebot. Wenn das Gesetz von Angebot und Nachfrage hier nicht spielt, dann liegt dies an der Intervention durch die Wirtschaftlichkeitsverfahren von santésuisse, wie wir im Folgenden zeigen wollen. Diese bewirken durch systematische Fehlbeurteilungen eine Fehllenkung der Behandlungspfade der ambulanten Psychiatrie, was gefährlich und teuer ist.

\section{Fehllenkung der Behandlungspfade}

Dass es mehr Zeit in Anspruch nimmt, einen Patienten psychotherapeutisch zu betreuen, als ihm nach kurzer Besprechung und/oder wenig Gesprächstherapie Psychopharmaka zu verschreiben, ist klar. Ein Arzt, der 
seine Patienten solcherart betreut, kann folglich auch weniger Patienten behandeln. Da Medikamente billiger sind als Gesprächstherapie, verursachen diese wenigen Patienten überdies durchschnittlich höhere Kosten. Solange der behandelnde Arzt dies mit Patienten ausgleichen kann, die weniger Therapie brauchen und mehr Medikation, erwachsen ihm daraus keine Schwierigkeiten. Sieht es aber so aus, dass sein Patientengut vorwiegend aus Patienten mit mittelschwerer Depression besteht, die er korrekterweise psychotherapeutisch behandelt, so können seine Durchschnittskosten schnell wesentlich höher sein als die von Kolleginnen und Kollegen. Vergleicht man etwa Praxen mit weniger als 100 Patienten pro Jahr mit solchen, welche 1000 und mehr Patienten behandeln, so ist die Vergleichbarkeit fragwürdig. Es können also bereits an der Patientenzahl Unterschiede in der Behandlungsart und im Grad der Patientenmorbidität abgelesen werden. Eine faire Beurteilung der Tätigkeit der psychiatrisch-psychotherapeutisch tätigen Ärztinnen und Ärzte sollte dies berücksichtigen und die Vergleichsgruppen entsprechend bilden. Das tun die Wirtschaftlichkeitsverfahren von santésuisse aber nicht. Mit gravierenden Folgen: Um sich aus der Gefahrenzone zu bringen, tendieren die behandelnden Ärzte eher zur Medikation, etwa in Situationen, da der Patient wenig Bereitschaft zeigt, sich auf eine Psychotherapie einzulassen, und es gälte, ihn dahingehend zu überzeugen. Dies ist denn auch das, was Felix Straumann in seinem Artikel feststellt, und es deckt sich auch mit der Tatsache, dass der Umsatz der entsprechenden Medikamente seit Jahren

Anzahl Behandelte Personen

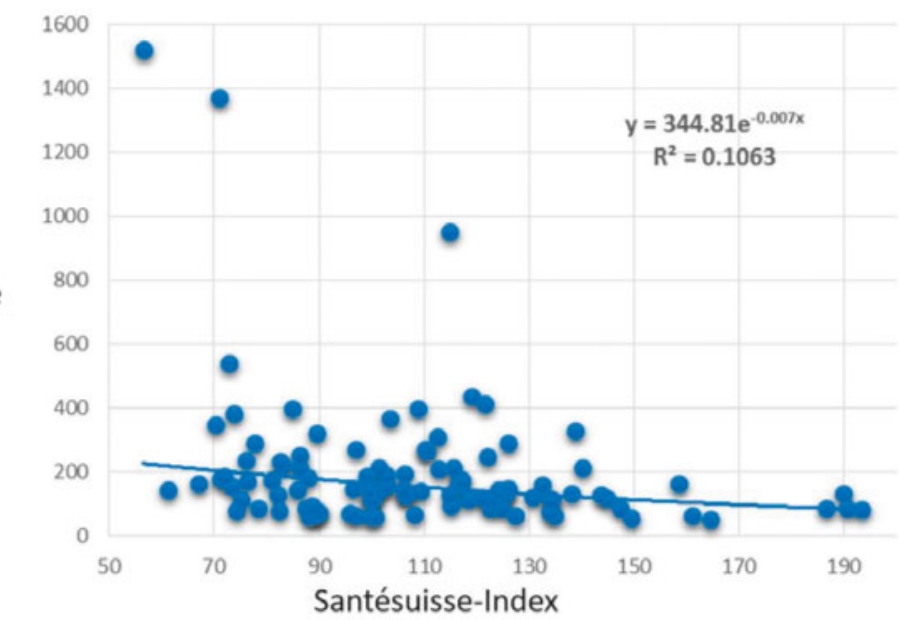

Abbildung 1: RSS-Indices und Anzahl Patienten der Vergleichsgruppe des Angeschuldigten (pro ZSR-Nummer, Jahr 2013, Psychiatrie, Kanton Aargau): auffällige Inhomogenität der Vergleichsgruppe mit deutlich niedrigerem Index bei hoher Patientenzahl (potentielle Unterversorgung). steigt [3]. Damit ist das Wirtschaftlichkeitsverfahren nun aber kein Beurteilungsverfahren mehr, sondern eine Intervention in die Behandlungspfade der Psychiatrie, welche auf diese Weise fehlgelenkt werden und Patienten mit leichter bis mittelschwerer Depression unter Umständen in eine lebenslange Medikamentenabhängigkeit oder gar in die Invalidität treiben können.

\section{Beispiel einer Fehlbeurteilung}

Im Februar dieses Jahres hat sich ein Arzt mit der Bitte an den VEMS gewandt, seinen Fall zu beurteilen. Er wurde bereits 2015 von santésuisse mit einem Verfahren belangt, aus welchem er sich, obwohl er sich keines Unrechts bewusst war, durch ein Schlichtungsergebnis der PVK mit einer Busse von 10000 CHF und Anwaltskosten von 20000 CHF befreien konnte (Akteneinsicht auf Anfrage). Wir vom VEMS haben immer wieder davor gewarnt, dass sich Ärztinnen und Ärzte, die korrekt arbeiten, mit solchen Vergleichen aus ihrem Verfahren herauskaufen, um nur endlich ihre Ruhe zu haben. Damit bestätigen sie falsche Einschätzungen durch santésuisse, womit sie nicht nur ihren Kolleginnen und Kollegen, sondern auch sich selbst einen Bärendienst erweisen. Da sie ihre «Schuld» nun ja eingestanden haben, kommen erneut Forderungen. Dies wiederfuhr auch dem betreffenden Arzt: Er wurde abermals belangt, und «in Anlehnung an das Schlichtungsergebnis der PVK» von 2015 wurde ihm auch gleich ein Vergleich angeboten, $15000 \mathrm{CHF}$, zahlbar in drei Monatsraten. Das ist ein zweifelhaftes Vorgehen. Entweder der Arzt hat überarztet, und dann ist er zu büssen, oder er hat es nicht, und dann sollte man ihn auch nicht in ein Verfahren ziehen. Ein Vergleich ist eine Einigung, und einigen kann man sich nicht vorsorglich, erst nach Abklärung der Positionen. Die Analyse der Vergleichsgruppendaten ergab, dass santésuisse den betreffenden Arzt, der weniger als 100 Patienten jährlich behandelt, mit Praxen vergleicht, die mehr als 1500 Patienten pro Jahr behandeln. Es zeigt sich dabei, dass grössere Praxen niedrige Rechnungsstellerstatistik-Indices (RSS-Indices) aufweisen (Abb. 1).

\section{Wirtschaftlichkeitsverfahren als Kosten- treiber}

In der Vergleichsgruppe des betreffenden Arztes haben wir eine Ausreisserpraxis, welche 1563 Patienten behandelt und einen RSS-Index von 56\% hat. Dies führt zu einer Verzerrung, die unter dem Begriff «Simpson Paradox» als statistisches Problem erkannt ist und an diesem besonders deutlichen Beispiel aufgezeigt werden soll. Das folgende Modell beruht auf der Modellierung fol- 
gender Kosten: Taxpunktwert 0,89 CHF, Taxpunkte pro 5 Minuten 17,9, Dauer der Sitzung 60 Minuten.

Wenn gemäss Kostengutsprache ein Patient mit 40 Sitzungen pro Jahr behandelt werden kann, ergeben sich Jahreskosten von 7646.90 CHF und somit bewilligte Jahreskosten von CHF 305875 (100\%, Praxis 1). Sieht der Psychiater die Patienten jedoch pro Jahr nur viermal für 60 Minuten, so entstehen Jahreskosten von 764.90 CHF pro Patient. Dies hat massive Effekte auf die Durchschnittskosten, insbesondere bei kleinen Praxen. Bereits 5 billige und 35 teure Patienten senken die Durchschnittskosten von $100 \%$ auf $89 \%$ (Praxis 2), in der umgekehrten Situation (Praxis 4) wäre der Index bei 21\% (siehe Tab. 1). Praxis 5 der Tabelle 1 behandelt beispielsweise 600 teure und 900 billige Patienten und kommt so auf $46 \%$ der Durchschnittskosten bei gleichzeitig massiver Belastung der Grundversicherung mit 5,3 Millionen Franken. Dass Praxis 5 ähnliche Morbiditäten behandelt wie Praxis 1, ist unmöglich. Wie will ein Arzt 600 Patienten 40 Mal pro Jahr während einer Stunde behandeln? Er müsste an 200 Arbeitstagen 26480 Stunden oder pro Tag 132,4 Stunden arbeiten. Tatsächlich hat santésuisse für die Ausreisserpraxis 5 aber 1,4 Millionen Franken Gesamtkosten ausgewiesen (Kostenindex 56\%). Es ist somit offensichtlich, dass diese ZSR-Nummer mehrere Ärzte einschliessen muss, und es ist davon auszugehen, dass in der Ausreisserpraxis 5 ganz andere Patientenmorbiditäten behandelt werden. Es wäre Aufgabe von santésuisse, zumindest auf der mathematischen Ebene plausible Vergleichspraxen herauszufiltern. Dieses Beispiel zeigt aber auch, wie die Wirtschaftlichkeitsverfahren zur Mengenausweitung führen können, was ihren Zweck gerade auf den Kopf stellt.

\section{Rechtsgrundlagen und Rechtspraxis}

Besonders stossend an obigem Fall sind zwei Dinge: Der betreffende Arzt wird für ein medizinisch korrek- tes Verhalten gebüsst, und er hat für all diese Behandlungen vorgängig Kostengutsprachen eingeholt und gutgeheissen bekommen. Es ist gemäss bundesgerichtlicher Auffassung falsch, wenn santésuisse bewilligte Kosten rückwirkend büssen will, weil sie im Vergleich mit Praxen, deren Arbeit nicht vergleichbar ist, höher liegen [4]. Aufgabe der Versicherer gemäss KVG ist es, Wirksamkeit, Zweckmässigkeit und Wirtschaftlichkeit der Behandlungen zu beurteilen. Wenn dabei Vergleiche mit anderen Leistungserbringern helfen sollen, dann müssen sie korrekt sein. Ist dies nicht möglich, so hat das Androhen von Klagen, vor allem wenn auch gleich ein Vergleich angeboten wird, den Charakter von Schutzgelderpressungen. Korrekt gälte es in solchen Situationen die Kosten auf der Ebene der Einzelfälle zu beurteilen, etwa indem die teuersten Patienten von einem unabhängigen medizinischen Fachgremium beurteilt würden. Weichen sie von den gutgesprochenen Beträgen ab, so kann Unzweckmässigkeit vorliegen, und dann wäre eine Busse berechtigt. Zeigt sich hingegen, wie dies hier der Fall ist, dass die Kosten eingehalten wurden, die Durchschnittskosten im Vergleich aber dennoch zu hoch sind, so ist der Vergleich zu hinterfragen und zu plausibilisieren. Mit der Unterlassung dieser Sorgfaltsregeln schafft santésuisse hingegen einen Anreiz, der die Kosten treibt und vermeidbares Leid schafft, indem er bewährte Behandlungspfade fehlleitet. Gemäss schweizerischer Verfassung Art. 29 Abs. 1 BV hat jede Person in Verfahren vor Gerichts- und Verwaltungsinstanzen Anspruch auf gleiche und gerechte Behandlung sowie auf Beurteilung innert angemessener Frist. Einen Teilgehalt dieses Verfahrensgrundrechts bildet auch der Anspruch auf Beurteilung durch eine unabhängige Behörde. In der OKP nimmt santésuisse eine behördliche Funktion subsidiär wahr und hat sich deshalb an die schweizerischen Standards zu halten. Im besagten Fall musste der Psychiater in den Vergleich einwilligen: $6000 \mathrm{CHF}$ war die Einigungssumme. Ein externer Experte hat

\begin{tabular}{|c|c|c|c|c|c|c|c|c|c|}
\hline & & \multicolumn{2}{|c|}{ Case Mix 1} & \multicolumn{2}{|c|}{ Case Mix 2} & \multicolumn{2}{|c|}{ Case Mix 3} & \multicolumn{2}{|c|}{ Case Mix 4} \\
\hline Praxistyp & Praxis 1 & Prax 2 & Prax 2 & Prax 3 & Prax 3 & Prax 4 & Prax 4 & Prax 5 & Prax 5 \\
\hline Taxpunktwert & 0.89 & 0.89 & 0.89 & 0.89 & 0.89 & 0.89 & 0.89 & 0.89 & 0.89 \\
\hline Taxpunkte pro 5 Minuten & 17.9 & 17.9 & 17.9 & 17.9 & 17.9 & 17.9 & 17.9 & 17.9 & 17.9 \\
\hline Anzahl 5 Minuten & 12 & 12 & 12 & 12 & 12 & 12 & 12 & 12 & 12 \\
\hline Kosten pro Sitzung & 191.17 & 191.17 & 191.17 & 191.17 & 191.17 & 191.17 & 191.17 & 191.17 & 191.17 \\
\hline Anzahl Sitzungen pro Jahr & 40 & 40 & 4 & 40 & 4 & 40 & 4 & 40 & 4 \\
\hline Jahreskosten pro Patient & 7647 & 7647 & 765 & 7647 & 765 & 7647 & 765 & 7647 & 765 \\
\hline Anzahl Patienten & 40 & 35 & 5 & 20 & 20 & 5 & 35 & 600 & 900 \\
\hline Praxisumsatz & 305875 & 267641 & 3823 & 152938 & 15294 & 38234 & 26764 & 4588128 & 688219 \\
\hline Summe & & & 271464 & & 168231 & & 64998 & & 5276347 \\
\hline Kosten/P & 7647 & & 6787 & & 4206 & & 1625 & & 3518 \\
\hline Durchschrittskosten & $100 \%$ & & $89 \%$ & & $55 \%$ & & $21 \%$ & & $46 \%$ \\
\hline
\end{tabular}

Tabelle 1: Casemix, Gesamtkosten, Prozent Durchschnittskosten in einem «Simpson Paradox»-Modell. 
den Fall kommentiert. Wir zitieren ihn hier, damit alle Psychiater gewarnt sind: «Das Problem ist, dass viele Psychiater in den letzten Jahren vermehrt mehr Sitzungszeiten und weniger Zeiten in Abwesenheit des Patienten verrechnen. Wer immer noch nach dem «alten» Muster - 50 Minuten Konsultation und $10 \mathrm{Mi}$ nuten in Abwesenheit des Patienten - abrechnet, wird so schnell auffällig. Im Klartext: Der betreffende Arzt rechnet wie ein Mediziner ab, nicht wie ein Ökonom, und das ist sein «Vergehen».

\section{Die Rolle der Fachgesellschaften}

Die Verbindung der psychiatrisch-psychotherapeutisch tätigen Ärztinnen und Ärzte der Schweiz FMPP hat das Problem denn auch erkannt. Sie schreibt ihren Mitgliedern: «Behandeln Sie also nicht Ihren Index, sondern Ihre Patienten" [5]. Dabei vertraut die FMPP auf die Vernunft von santésuisse: «Sofern Sie Ihre Patienten nach bestem Wissen und Gewissen und unter korrekter Anwendung der gesetzlichen Bestimmungen behandeln, hat santésuisse aufgrund der bestehenden Rechtslage mit Sicherheit kein Interesse daran, einen Gerichtsprozess zu führen, da ihr Prozessrisiko viel zu hoch ist.» Das ist eingedenk der Praxis von santésuisse und des erwähnten Beispiels ein doch recht gefährlicher Rat. Hier kann ein einzelner Arzt wenig ausrichten. Die FMPP sollte deshalb nicht ihren Mitgliedern mit fragwürdigen Tipps ein gefährliches Gefühl der Sicherheit vermitteln, sondern vielmehr entschieden intervenieren und auf Verbandsebene gegen das Vorgehen von santésuisse Schritte unternehmen, insbesondere eingedenk der Tatsache, dass sich auch die Arbeitsgruppe WZW (Wirksamkeit, Zweckmässigkeit, Wirtschaftlichkeit) von FMH und santésuisse seit Jahren im Kreis dreht. Von dieser Seite erwarten wir seit mehr als einem halben Jahr ein externes Gutachten mit Vorschlägen zur Verfeinerung der Methode. Der VEMS ist im Kontakt mit der Leitung der Arbeitsgruppe und wird bis heute vertröstet (man plane, gemäss E-Mail von Thomas Kessler vom 4.12.2017, nun das weitere Vorgehen «bis ins Jahr 2023»).

\section{Mängel und Verbesserungsvorschläge}

Dringender Verbesserungsbedarf besteht in den durch Fachgesellschaften definierten predictive ratios, in der Erfassung der Behandlungskosten pro Jahr (und nicht der Rechnungsstellungskosten pro Jahr), in der Plausibilisierung der SASIS-Grunddaten, in der Verbesserung der Erstellung der häufig rein willkürlichen Vergleichsgruppen, in der Präzisierung der Patientenprofile anhand von PCG (pharmaceutical cost groups), in der Offenlegung der fachlichen Kompetenzen für die Analyse der Praxisbesonderheiten, in der Offenlegung der vertraglichen Vereinbarungen der Prüfer mit santésuisse, in der Professionalisierung der Phase der analytischen Methode (Praxisbesonderheiten) durch die Fachgesellschaften, im konsequenten Entfernen von Kosten, für welche eine Kostengutsprache existiert (dann ist auch in der Regel ein Vergleich mit einer Vergleichsgruppe ohnehin hinfällig), und in der Erfassung der Kosten pro GLN-Nummer anstelle der ZSR-Nummer. Es gehört zum Patientenauftrag, sich um diese Verbesserungen $\mathrm{zu}$ bemühen, denn die derzeitige Prüfmethode gefährdet, wie oben aufgezeigt wurde, das Patientenwohl in gefährlicher Weise und treibt überdies die Kosten.

\section{Bildnachweis}

Grafik zur Verfügung gestellt von den Autoren

\section{Literatur}

1 Siehe Behandlungsempfehlungen SGPP.

2 http://www.tagesanzeiger.ch/wissen/medizin-und-psychologie/ den-therapeuten-am-draht/story/17201297

3 Siehe Helsana Arzneimittelreport 2017.

4 Siehe VEMS-Gutachten Prof. Ueli Kieser: http://www.docfind.ch/ KieserGutachten0617.pdf

5 http://www.docfind.ch/WZWMerkblattFMPP062016.pdf 